\title{
Posterior condylar offset changes and its effect on clinical outcomes after posterior- substituting, fixed-bearing total knee arthroplasty: anterior versus posterior referencing
}

Moon Jong Chang ${ }^{1}$, Seung-Baik Kang ${ }^{1 *} \mathbb{D}$, Chong Bum Chang ${ }^{1}$, Do Hwan Han'1, Hyung Jun Park', Keummin Hwang ${ }^{1}$, Jisu Park², II-Ung Hwang ${ }^{3}$, Seung Ah. Lee ${ }^{4}$ and Sohee Oh ${ }^{5}$

\begin{abstract}
Background: We sought to determine whether there was a difference in the posterior condylar offset (PCO), posterior condylar offset ratio (PCOR) and clinical outcomes following total knee arthroplasty (TKA) with anterior referencing (AR) or posterior referencing (PR) systems. We also assessed whether the PCO and PCOR changes, as well as patient factors were related to range of motion (ROM) in each referencing system.

Methods: This retrospective study included 130 consecutive patients (184 knees) with osteoarthritis who underwent primary posterior cruciate ligament (PCL)-substituting fixed-bearing TKA. The difference between preoperative and postoperative PCO and PCOR values were calculated. Clinical outcomes including ROM and Western Ontario and McMaster University (WOMAC) scores were evaluated. Furthermore, multiple linear regression analysis was performed to determine the factors related to postoperative ROM in each referencing system.

Results: The postoperative PCO was greater in the AR group $(28.4 \mathrm{~mm})$ than in the PR group $(27.4 \mathrm{~mm})$, whereas the PCO was more consistently preserved in the PR group. The mean postoperative ROM after TKA was greater in the AR group $\left(129^{\circ}\right)$ than in the PR group $\left(122^{\circ}\right)$, whereas improvement in WOMAC score did not differ between the two groups. Preoperative ROM was the only factor related to postoperative ROM in both groups.

Conclusions: There was no difference in postoperative PCO in AR and PR group and the PCO was not associated with postoperative ROM. PCO was more consistently preserved after surgery in the PR group. The postoperative PCO and PCOR changes did not affect the postoperative ROM. Furthermore, similar clinical outcomes were achieved in the AR and PR groups.
\end{abstract}

Trial registration: Retrospectively registered (Trial registration number: 06-2010-110).

Keywords: Anterior referencing system, Posterior referencing system, Posterior condylar offset, Posterior condylar offset ratio

\footnotetext{
* Correspondence: ossbkang@gmail.com

1 Department of Orthopaedic Surgery, Seoul National University College of

Medicine, SMG-SNU Boramae Medical Center, Seoul 07061, South Korea

Full list of author information is available at the end of the article
}

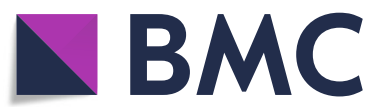

Part of Springer Nature (c) The Author(s). 2020 Open Access This article is distributed under the terms of the Creative Commons Attribution 4.0 International License (http://creativecommons.org/licenses/by/4.0/), which permits unrestricted use, distribution, and reproduction in any medium, provided you give appropriate credit to the original author(s) and the source, provide a link to the Creative Commons license, and indicate if changes were made. The Creative Commons Public Domain Dedication waiver (http://creativecommons.org/publicdomain/zero/1.0/) applies to the data made available in this article, unless otherwise stated. 


\section{Background}

Proper implant positioning and sizing are crucial for successful total knee arthroplasty (TKA) [1-4]. Anterior referencing (AR) and posterior referencing (PR) systems are the two major systems for positioning and sizing of the femoral component. The AR system has the reduced risk of femoral notching or patellofemoral overstuffing, whereas posterior condylar resection is not set. In contrast, the posterior condylar resection is set in the PR system, whereas the anterior femoral cutting is less predictable [5]. Therefore, each referencing system has its own advantages and disadvantages.

However, in contemporary TKA, traditional concepts of the AR or PR system might not be as certain $[5,6]$. Theoretical disadvantages of each referencing system could be overcome by meticulous surgical techniques, advanced surgical instruments and newly developed implants. Some recent implant systems adopt the femoral component that has a smaller mediolateral dimension. In PS knee, because the PCL is removed, the flexion gap is often widened. Thus, if there is increased flexion gap during surgery in the AR system, it could be compensated with by up-sizing the femoral component without mediolateral overhang. In addition, there are implants that have a femoral component with an increased cutting angle of the anterior flange to avoid AFC notching, and thus, down-sizing the femoral component is possible in the PR system.

On the other hand, preservation of the posterior condylar offset $(\mathrm{PCO})$ and posterior condylar offset ratio (PCOR) was reported to be related to the degree of maximal flexion after TKA [7-16]. The PCO or PCOR after TKA could differ based on the referencing system used. Theoretically, the PCO or PCOR can be consistently preserved in the PR system because the same amount of resected bone is replaced by the femoral component. Thus, it is reasonable to speculate that the PR system could lead to greater flexion after TKA. However, besides the PCO and PCOR, there are many factors that could be related to range of motion (ROM) after TKA, such as preoperative ROM, posterior slope of the tibial component and the type of implant used $[14,17,18]$. In addition, the factors related to ROM after surgery could differ between the AR and PR systems. However, there is no consensus that exist regarding these issues [11-13, 19].

Therefore, we sought to determine whether there was a difference in PCO and PCOR after TKA between the AR and PR systems. We also attempted to determine whether PCO and PCOR changes, as well as patient factors, were related to ROM after TKA using each referencing system. In addition, we aimed to examine whether the improvement of clinical outcomes after TKA differed between the two referencing systems. We hypothesized that restoration of the PCO and PCOR after TKA would be better in the PR system than the AR system, and that changes in the PCO and PCOR would be related to ROM after TKA using both referencing systems. Finally, we hypothesized that the clinical outcome after TKA using the PR system would be better than that using AR system.

\section{Methods}

This was a retrospective study that started with 154 patients, who underwent primary TKA using the same prosthesis due to end-stage osteoarthritis (OA) at our institution, from February 2014 to November 2015. Of these, 16 were excluded because of a history or objective evidence of rheumatoid arthritis [5], posttraumatic arthritis [6], concomitant neuronal disease [3], weakness or severe instability in the operated limb [2]. Patients who with less than 2 years of follow-up were also excluded [8]. Consequently, 130 patients (184 knees) with OA who underwent primary TKA were included in this study. There were 118 females (91\%) and 12 (9\%) males, with a mean age of 72 years old (standard deviation [SD], 6.2 years; range, 57 to 88 years). The mean preoperative height and weight were $152.2 \mathrm{~cm}$ (SD, 6.0; range, 138 to $173.9 \mathrm{~cm}$ ) and $62 \mathrm{~kg}(\mathrm{SD}, 8.7$; range, 33 to $84 \mathrm{~kg}$ ). The mean body mass index (BMI) was $26.7 \mathrm{~kg} /$ $\mathrm{m} 2$ (SD, 3.4; range, 16.8 to $36.6 \mathrm{~kg} / \mathrm{m} 2$ ). The median follow-up was 37 months (range, 26 to 47 months). Patients were categorized into two groups according to the referencing system used. There were no differences in sex, BMI and laterality of the operated limb between the two groups, even though the mean age was higher in the AR group $(p=0.008)$ (Table 1$)$. The study protocol was approved by the institutional review board of authors' hospital (IRB number: 06-2010-110).

All surgeries were performed by a single senior surgeon using the same surgical techniques, except for the referencing system (AR or PR system) used. An anterior

Table 1 Comparisons of the patient demographics and preoperative variables between the AR and the PR groups

\begin{tabular}{llll}
\hline Variable & $\begin{array}{l}\text { AR group } \\
(n=93)\end{array}$ & $\begin{array}{l}\text { PR group } \\
(n=91)\end{array}$ & $p$-value \\
\hline Sex, Female & $64(94 \%)$ & $54(87 \%)$ & 0.550 \\
Age (years) & $73 \pm 6.1$ & $71 \pm 6.0$ & 0.008 \\
BMI $\left(\mathrm{kg} / \mathrm{m}^{2}\right)$ & $26.5 \pm 3.6$ & $26.5 \pm 3.6$ & 0.494 \\
PCO $(\mathrm{mm})$ & $28.1 \pm 3.6$ & $27.4 \pm 2.8$ & 0.126 \\
PCOR & $0.47 \pm 0.04$ & $0.47 \pm 0.03$ & 0.394 \\
ROM $\left(^{\circ}\right)$ & $120 \pm 17.1$ & $120 \pm 16.6$ & 0.804 \\
WOMAC & $44.2 \pm 15.9$ & $38.0 \pm 12.6$ & 0.038 \\
\hline
\end{tabular}

Data are presented as the means and standard deviations (SD) Abbreviations: $A R$ anterior referencing, $P R$ posterior referencing, $P C O$ posterior condylar offset, $P C O R$ posterior condylar offset ratio, $R O M$ range of motion, WOMAC Western Ontario and McMaster University osteoarthritis index, BMI body mass index 
midline incision and medial parapatellar arthrotomy was performed with a tourniquet applied. The posterior cruciate ligament (PCL) was substituted with a fixedbearing implant. All knees were implanted with the U2 ${ }^{\circ}$ Total Knee System (United, Taipei, Taiwan). All femoral, tibial, and patellar components were cemented. When using the AR system, the stylus was positioned in the highest position of lateral aspect of anterior femur to determine the anterior cutting level. Anterior femoral cutting was performed to a perfect match for the anterior cortical line. A larger femoral component was chosen to prevent an increased flexion gap while using PCLsubstituting implant if the femoral component was measured between two sizes (Table 2). In contrast, when using the PR system, preliminary anterior resection using the cutting zig of the up-sized femoral component was performed to assess the possibility of AFC notching. Then, the down-sized component was selected if possible to prevent overstuffing of patellofemoral joint. We aimed to cut the proximal tibia at a 0 -degree slope in all patients.

We performed radiographic evaluations using a picture archiving and communication system (PACS) (MaroviewTM, Marotech, Seoul, Korea). The conventional radiographs taken preoperatively and at the last follow-up visit were used. True pre- and postoperative lateral radiographs, with perfect overlap of the medial and lateral femoral condyles, were used in all patients. The PCO was evaluated on the lateral radiographs by measuring the maximum distance between the tangent of the femoral diaphysis posterior cortex and the posterior condylar margin (Fig. 1) [14]. The PCOR was calculated by dividing the $\mathrm{PCO}$ by the maximum distance between the posterior condylar border and the tangent of the femoral diaphysis anterior cortex (Fig. 1) [20]. The preoperative data showed no differences in terms of mean PCO and PCOR between the AR and PR groups ( $p=0.126$ and $p=0.398$, respectively) (Table 1$)$. Interand intra-observer reliabilities for the PCO and PCOR measurements were performed using intra-class correlation coefficients (ICC). The two observers repeated the measurements two times within a 2-week interval. The ICCs of the intra-observer reliabilities (PCO, 0.94;

Table 2 Proportion of femoral component size selected in each referencing system

\begin{tabular}{lll}
\hline Variable & $\begin{array}{l}\text { AR group } \\
(n=93)\end{array}$ & $\begin{array}{l}\text { PR group } \\
(n=91)\end{array}$ \\
\hline Size as it is & $49(53 \%)$ & $71(78 \%)$ \\
Up-sized & $39(42 \%)$ & $3(3 \%)$ \\
Down-sized & $5(5 \%)$ & $17(19 \%)$ \\
\hline
\end{tabular}

Data are presented as the numbers of patients, with the proportions in parentheses. Abbreviations: $A R$ Anterior referencing, $P R$ posterior referencing
PCOR, 0.86) and inter-observer reliabilities (PCO, 0.89; PCOR, 0.83) were satisfactory.

Clinical outcomes were evaluated by an independent clinical investigator preoperatively and 2 years postoperatively. The presence of a flexion contracture and maximum flexion, in a supine position, were measured using a goniometer. Western Ontario and McMaster University (WOMAC) scores were used to evaluate the clinical outcome after surgery.

Statistical analyses were performed using SPSS for Windows (version 19.0; SPSS Inc., Chicago, IL), and $p$ values of $<0.05$ were considered statistically significant. Demographic and clinical data such as age, BMI, PCO, PCOR, ROM and WOMAC score were described as means and standard deviations (SD). Continuous variables between the two groups were compared using the Student's t-test. The proportions of the categorical variables in the two groups were compared by using the chi-square test. The change between pre- and postoperative values was determined using the paired $t$-test.

To evaluate how well the PCO and PCOR were preserved in each referencing system, the differences between pre-and postoperative PCO values were classified into 3 groups: group $1(\leq-2 \mathrm{~mm})$, group $2(-2$ $\mathrm{mm}<$ PCO difference $\leq 2 \mathrm{~mm})$, and group $3(>2 \mathrm{~mm})$. In addition, the differences between the pre-and postoperative PCOR values were also classified into 3 groups: group I $(\leq-0.03)$, group II $(-0.03<$ PCOR difference $\leq$ $0.03)$, and group III $(>0.03)$. The reference value $(2 \mathrm{~mm})$ for the PCO difference was derived from a previous study that found the mean cartilage thickness of femur to be $2.2 \mathrm{~mm}$ [21]. We assumed that $\pm 2 \mathrm{~mm}$ difference of the $\mathrm{PCO}$ is an acceptable change considering that a plain radiograph cannot account for the thickness of the cartilage. In addition, Johal et al. mentioned that a mean difference of 0.03 for the PCOR was observed after TKA, so we postulated that a PCOR difference of more than 0.03 after surgery was meaningful [22]. To determine the statistical significance of the difference in the proportions of PCO and PCOR changes between the two groups, the Chi-square test was used. In addition, the one-way analysis of variance (ANOVA) was used to determine whether there was a difference in postoperative ROM among the three groups, which was divided by the amount of changes in the PCO and PCOR after surgery. The multiple linear regression analysis using variable with $p<0.05$ was performed to determine the factors that were related with postoperative ROM. Independent variables included age, BMI, sex, tibial slope and PCO changes and preoperative ROM.

\section{Results}

The postoperative mean PCO value was $28.4 \mathrm{~mm}$ in the AR group and $27.4 \mathrm{~mm}$ in the PR group $(p=0.038)$, 


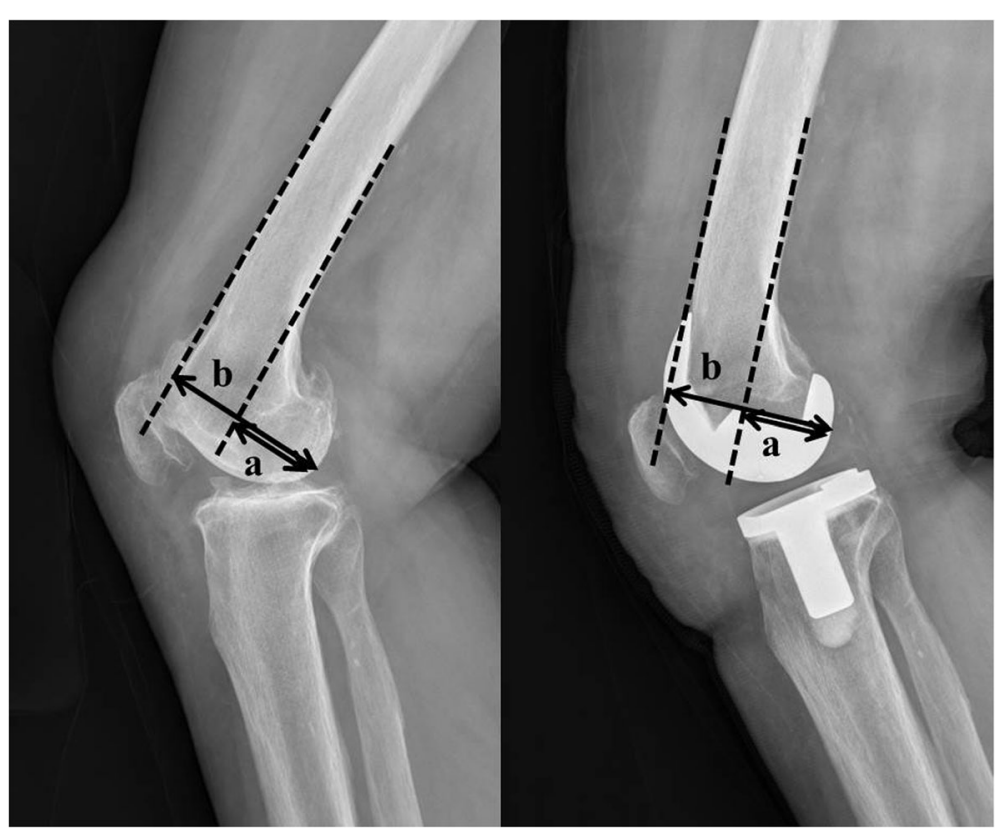

Fig. 1 Measurement of the posterior condylar offset (PCO, a) and posterior condylar offset ratio (PCOR, a/b) based on true lateral preoperative and postoperative knee radiographs. a distance in millimeters from the tangent of the femoral diaphysis posterior cortex to the posterior condylar margin; $\mathbf{b}$ distance in millimeters from the posterior condylar border to the tangent of the femoral diaphysis anterior cortex

whereas the PCO values were more consistently preserved in the PR group. And there was no difference in the mean postoperative PCOR values between the two groups $(p=0.392)$. In terms of the mean amount of changes in the pre- and post-TKA PCO and PCOR, there was no significant difference between the two groups ( $p=0.567$ and $p=0.988$, respectively) (Table 3 ). However, the proportion of the knees with a relatively well preserved PCO $(-2 \mathrm{~mm}<\mathrm{PCO}$ difference $\leq 2 \mathrm{~mm})$ was higher in the $\mathrm{PR}$ group than the $\mathrm{AR}$ group $(p=0.039)$ (Fig. 2). And there was no significant notching case like violation of the outer and the inner table of the anterior femoral cortex in down-sizing PR group $(n=17)$.

Table 3 Comparisons of the postoperative variables between the $A R$ and the PR groups

\begin{tabular}{llll}
\hline Variable & $\begin{array}{l}\text { AR group } \\
(n=93)\end{array}$ & $\begin{array}{l}\text { PR group } \\
(n=91)\end{array}$ & -value \\
\hline PCO $(\mathrm{mm})$ & $28.4 \pm 3.5$ & $27.4 \pm 2.8$ & 0.038 \\
PCOR & $0.47 \pm 0.04$ & $0.47 \pm 0.04$ & 0.392 \\
PCO changes $(\mathrm{mm})$ & $0.22 \pm 3.6$ & $0 \pm 2.4$ & 0.567 \\
PCOR changes & $0 \pm 0.37$ & $0 \pm 0.25$ & 0.988 \\
ROM $\left({ }^{\circ}\right)$ & $129 \pm 4.6$ & $122 \pm 14.3$ & $<0.001$ \\
WOMAC & $22.3 \pm 9.8$ & $17.1 \pm 9.6$ & 0.001 \\
\hline
\end{tabular}

Data are presented as the means and standard deviations (SD)

Abbreviations: $A R$ anterior referencing, $P R$ posterior referencing, $P C O$ posterior condylar offset, $P C O R$ posterior condylar offset ratio, $R O M$ range of motion, WOMAC Western Ontario and McMaster University osteoarthritis index
There was no difference in postoperative ROM, according to the amount of PCO and PCOR changes in the AR group ( $p=0.935$ and 0.688$)$ and the PR group $(p=0.940$ and 0.552$)$ (Fig. 3). The preoperative ROM was the only factor related to the postoperative ROM in both groups (Tables 4 and 5 ).

The mean postoperative ROM and its improvement after TKA were greater in the AR group than the PR group, whereas improvement in WOMAC score did not differ between the two groups. The mean postoperative ROM was significantly greater in the AR group $\left(129^{\circ}\right)$ than in the PR group $\left(122^{\circ}\right)(p<0.001)$ (Table 3). Moreover, the proportional changes in ROM after TKA was also significantly higher in the AR group $\left(9^{\circ}\right)$ than in the PR group $\left(2^{\circ}\right) \quad(p=0.007)$. The postoperative mean WOMAC was better in the PR group than in the AR groups (17.1 vs. 22.3, $p=0.001$ ) (Table 3 ). However, the change in WOMAC after TKA showed no difference between the two groups $(p=0.205)$.

\section{Discussion}

The postoperative PCO and PCOR have been shown to be related to postoperative ROM, although results have been controversial $[8,12-14]$. Furthermore, the postoperative PCO or PCOR can be affected based on the referencing system used. The principal findings of this study were: 1 ) There was no difference in postoperative $\mathrm{PCO}$ in AR and PR group and the PCO was more consistently preserved in the PR group; 2) changes in PCO 

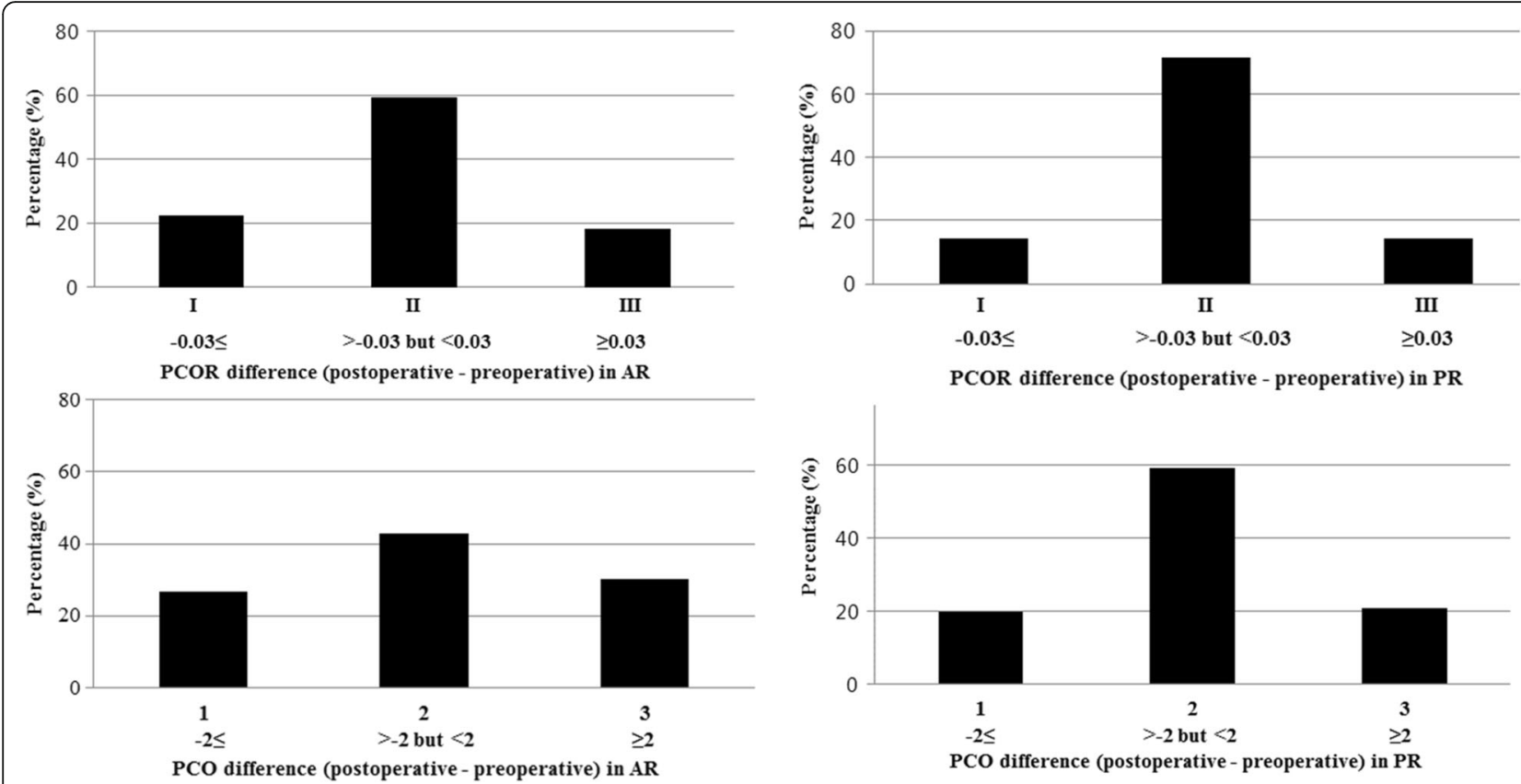

Fig. 2 Proportional differences of the knees (\%) between pre- and post-TKA based on the posterior condylar offset (PCO) and posterior condylar offset ratio (PCOR). The proportion of the preserved PCO $(-2 \mathrm{~mm}<\mathrm{PCO}$ difference $\leq 2 \mathrm{~mm})$ was higher in the PR group than the AR $\operatorname{group}(p=0.039)$

and PCOR after surgery were not associated with postoperative ROM in the AR and PR groups, and the only related factor for the postoperative ROM was the preoperative $\mathrm{ROM}$ regardless of referencing system used; 3) although the postoperative ROM was better in the AR group, there was no difference in improvement of WOMAC scores after surgery between the AR and PR groups.

Our findings did not completely support the hypothesis that restoration of the PCO and PCOR would be better in the PR group compared to the AR group. The portion of knees with a well-preserved PCO was higher in the PR group. However, the mean postoperative PCO was greater in the AR group. Almeida et al. reported that there was no difference in the postoperative $\mathrm{PCO}$ and PCOR between the AR and PR groups [7]. In the previous study, the mean postoperative PCO was 27.4 $\mathrm{mm}$ in the AR group and $27.7 \mathrm{~mm}$ in the PR group ( $p=$ 0.32 ). Although the implants used in the previous study were different than those used in this study, the results of the postoperative PCO and POCR values were similar. Furthermore, the previous study compared the medial and lateral PCO values between AR and PR systems using computed tomography, which revealed that the PCO values after TKA were greater in the AR group than in the PR group [11]. This study selected a larger implant for femurs in-between sizes to prevent flexion instability. Therefore, the proportion of up-sized femoral component was higher in the AR group, and this could affect the greater postoperative mean $\mathrm{PCO}$ in the AR group (Table 2). Therefore, our findings suggest that the postoperative PCO and PCOR could be preserved in both the AR and PR systems using a contemporary surgical technique and implant.

In the current literature, it is controversial whether the postoperative PCO and PCOR are related to postoperative ROM. Initially, Bellemans et al. suggested the concept of the PCO, and they found the relationship between the postoperative PCO and postoperative ROM in TKA performed using a PCL-retaining implant [8]. The previous study explained the cause of ROM limitations using the phenomenon of early impingement between the posterior cortex of the femur and the posterior lip of the polyethylene insert. This finding was caused by several factors, such as a paradoxical roll forward with flexion, a reduced posterior slope of the tibial component and a high posterior lip in the polyethylene insert [12]. Malviay et al. showed similar findings in patients with TKA using a PCL-retaining implant, and the postoperative PCO had the greatest impact on the final ROM [14]. There was significant correlation between the postoperative PCO and ROM at 12 months after surgery $(\mathrm{r}=0.65 ; p<0.0001)$. However, the relationship between the postoperative PCO and postoperative ROM was not consistently reproduced in the following studies using PCL-substituting or cruciate-sacrificing implants $[12,13]$. In the present study, there was no significant correlation between differences in the PCO or 

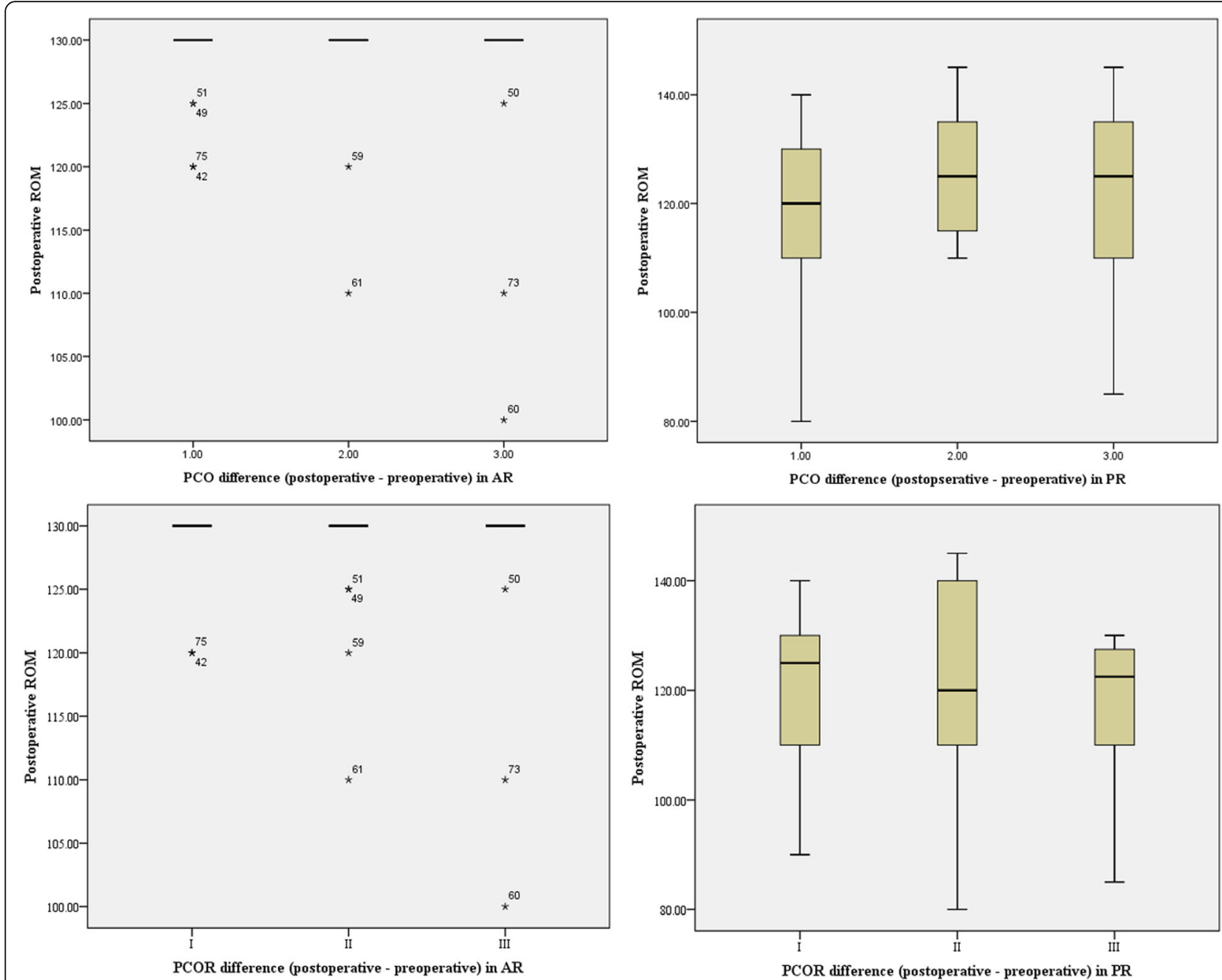

Fig. 3 Postoperative range of motion, according to the difference between pre- and post-TKA based on posterior condylar offset (PCO) and posterior condylar offset ratio (PCOR). Postoperative ROM did not differ regardless of PCO and PCOR differences in both the AR ( $p=0.935$ and 0.688) and the PR group ( $p=0.940$ and 0.552$)$

Table 4 Factors related to the postoperative range of motion in the AR group

\begin{tabular}{|c|c|c|c|c|}
\hline \multirow[t]{2}{*}{ Variable } & \multicolumn{2}{|c|}{ Univariable analysis } & \multicolumn{2}{|c|}{ Multivariable analysis } \\
\hline & $\beta$ & $p$-value & $\beta$ & $p$-value \\
\hline Age & -0.004 & 0.955 & & \\
\hline Sex, Female & 0.460 & 0.814 & & \\
\hline BMl & 0.052 & 0.700 & & \\
\hline Preoperative ROM & 0.078 & 0.009 & 0.078 & 0.009 \\
\hline Tibial slope & 0.098 & 0.654 & & \\
\hline PCO changes & -0.184 & 0.160 & & \\
\hline Adjusted $R^{2}$ & & & & 0.067 \\
\hline
\end{tabular}

Data are presented as the $p$-values and regression coefficients $(\beta)$ Abbreviations: $A R$ anterior referencing, $B M I$ body mass index, $R O M$ range of motion, $P C O$ posterior condylar offset
PCOR and postoperative ROM, regardless of referencing systems used. The contradictory findings of our study to those of Bellemans et al. may be explained in part by the fact that we used a different design of the PCLsubstituting implant.

Alexander et al. demonstrated that there are no statistically significant differences in surgical or clinical outcomes between the AR and PR systems. The mean Knee Society Score at 2 years after TKA was 98.11 in the AR group and 97.69 in the PR group $(p=0.7647,5)$. In our study, the magnitude of improvement in WOMAC score after surgery showed no difference between the two groups. On the other hand, both the mean postoperative ROM and improvement of ROM were greater in AR group. However, both groups achieved a mean postoperative ROM greater than $120^{\circ}$. If a flexion arc of $110^{\circ}$ or more is achieved, improvement of activities in daily life 
Table 5 Factors related to the postoperative range of motion in the PR group

\begin{tabular}{|c|c|c|c|c|}
\hline \multirow[t]{2}{*}{ Variable } & \multicolumn{2}{|c|}{ Univariable analysis } & \multicolumn{2}{|c|}{ Multivariable analysis } \\
\hline & $\beta$ & $p$-value & $\beta$ & $p$-value \\
\hline Age & -0.142 & 0.589 & & \\
\hline Sex, Female & -2.500 & 0.640 & & \\
\hline BMI & -0.989 & 0.045 & -0.733 & 0.123 \\
\hline Preoperative ROM & 0.292 & 0.001 & 0.269 & 0.003 \\
\hline Tibial slope & -0.263 & 0.816 & & \\
\hline PCO changes & 0.322 & 0.614 & & \\
\hline Adjusted $R^{2}$ & & & & 0.123 \\
\hline
\end{tabular}

Data are presented as the $p$-values and regression coefficients $(\beta)$ Abbreviations: $P R$ posterior referencing, $B M I$ body mass index, $R O M$ range of motion, $P C O$ posterior condylar offset

can be expected [23]. Combining the findings of previous studies and this study, the AR and PR systems would both contribute to the comparable clinical outcomes after TKA using a PCL-substituting fixed-bearing implant.

This study has several limitations. First, the study was retrospectively performed and AR and PR group depends on man's preference, this can lead to selection bias.. Second, a true lateral radiograph was used to assess the PCO and PCOR, which cannot reflect cartilage thickness. However, all pre- and post-TKA radiographic parameters were measured twice, and intra- and interobserver measurement reliabilities showed good to excellent agreement. This suggests that the PCO and PCOR values used in this study were reliable. Third, the results may not be generalized to all TKA patients because of the various implant designs. However, this study provides valuable information to readers by comparing two referencing systems in the same PCLsubstituting fixed-bearing implant. Fourth, The evaluation of the condyles of the femur on plain lateral X-ray has limitations on evaluating each medial and lateral condyle of femur accurately. However, the accurate evaluation by CT is also difficult due to the influence of the metal artifact. Moreover, in many previous studies, plain X-ray was mainly used for evaluation, so it was easier to compare with other studies. Also, Evaluating patients with CT for the study is not ethical. So we did research using X-ray. Finally, the follow-up period of two years was relatively short. However, the two-year follow-up was enough to present postoperative ROM and knee scores because the postoperative ROM rarely changes one year after surgery [24].

\section{Conclusions}

There was no difference in postoperative $\mathrm{PCO}$ in AR and PR group and the PCO was more consistently preserved after surgery in the PR group. The postoperative
PCO and PCOR changes did not affect the postoperative ROM, regardless of the referencing system used after PCL-substituting fixed-bearing TKA. Furthermore, comparable satisfactory clinical outcomes were achieved in both groups.

\section{Abbreviations}

AR: Anterior referencing system; PCO: Posterior condylar offset:

PCOR: Posterior condylar offset ratio; PR: Posterior referencing system

Acknowledgements

Not applicable.

\section{Authors' contributions}

All authors were involved equally in all aspects of the creation of this manuscript. All authors read and approved the final manuscript.

\section{Funding}

This research was supported by the Bio \& Medical Technology Development Program of the National Research Foundation (NRF) funded by the Ministry of Science \& ICT (2017M3A9D8063538).

\section{Availability of data and materials}

The datasets used or analysed during the current study are available from the corresponding author on reasonable request.

Ethics approval and consent to participate

This study was approved in SMG-SNU BMC IRB (No. 06-2010-110).

Consent for publication

Not applicable.

\section{Competing interests}

The authors declare that they have no competing interests.

\section{Author details}

${ }^{1}$ Department of Orthopaedic Surgery, Seoul National University College of Medicine, SMG-SNU Boramae Medical Center, Seoul 07061, South Korea. ${ }^{2}$ Department of Orthopaedic Surgery, Seoul National University College of Medicine, Seoul National University Hospital, Seoul 03080, South Korea. ${ }^{3}$ Department of Emergency Medicine, Seoul National University College of Medicine, Seoul National University Hospital, Seoul 03080, South Korea.

${ }^{4}$ Department of Physical Medicine and Rehabilitation, College of Medicine, Kyung Hee University, Seoul 05278, South Korea. ${ }^{5}$ Department of Biostatistics, SMG-SNU Boramae Medical Center, Seoul 07061, South Korea.

Received: 7 August 2019 Accepted: 21 November 2019

Published online: 01 January 2020

\section{References}

1. Angibaud LD, Dai Y, Liebelt RA, Gao B, Gulbransen SW, Silver XS (2015) Evaluation of the accuracy and precision of a next generation computerassisted surgical system. Clin Orthop Surg 7(2):225-233

2. Incavo SJ, Beynnon BD, Johnson CC, Churchill DL (1997) Knee kinematics in genesis total knee arthroplasty. A comparison of different tibial designs with and without posterior cruciate substitution in cadaveric specimens. Am J Knee Surg 10(4):209-215

3. Lee D-H, Lee S-H, Song E-K, Seon J-K, Lim H-A, Yang H-Y (2017) Causes and clinical outcomes of revision total knee arthroplasty. Knee Surg Related Res 29(2):104

4. Lee JH, Wang S-I (2015) Risk of anterior femoral notching in navigated total knee arthroplasty. Clin Orthop Surg 7(2):217-224

5. Fokin AA, Heekin RD (2014) Anterior referencing versus posterior referencing in total knee arthroplasty. J Knee Surg 27(04):303-308

6. Manson TT, Khanuja HS, Jacobs MA, Hungerford MW (2009) Sagittal plane balancing in the total knee arthroplasty. J Surg Orthop Adv 18(2):83-92

7. Almeida P, Vilaça A (2015) The posterior condylar offset ratio and femoral anatomy in anterior versus posterior referencing total knee arthroplasty. Orthop Traumatol Surg Res 101(6):687-691 
8. Bellemans J, Banks S, Victor J, Vandenneucker H, Moemans A (2002) Fluoroscopic analysis of the kinematics of deep flexion in total knee arthroplasty: influence of posterior condylar offset. J Bone Joint Surg 84(1):50-53

9. Clement N, MacDonald D, Hamilton D, Burnett R (2017) Posterior condylar offset is an independent predictor of functional outcome after revision total knee arthroplasty. Bone Joint Res 6(3):172-178

10. Goldstein WM, Raab J, Gleason TF, Branson JJ, Berland K (2006) Why posterior cruciate-retaining and substituting total knee replacements have similar ranges of motion: the importance of posterior condylar offset and cleanout of posterior condylar space. JBJS. 88:182-188

11. Han H, Oh S, Chang CB, Kang S-B (2016) Changes in femoral posterior condylar offset and knee flexion after PCL-substituting total knee arthroplasty: comparison of anterior and posterior referencing systems. Knee Surg Sports Traumatol Arthrosc 24(8):2483-2488

12. Hanratty B, Thompson N, Wilson R, Beverland D (2007) The influence of posterior condylar offset on knee flexion after total knee replacement using a cruciate-sacrificing mobile-bearing implant. J Bone Joint Surg 89(7):915-918

13. Ishii Y, Noguchi H, Takeda M, Sato J, Toyabe S-i (2013) Posterior condylar offset does not correlate with knee flexion after TKA. Clin Orthop Relat Res 471(9):2995-3001

14. Malviya A, Lingard E, Weir D, Deehan D (2009) Predicting range of movement after knee replacement: the importance of posterior condylar offset and tibial slope. Knee Surg Sports Traumatol Arthrosc 17(5):491-498

15. Massin P, Gournay A (2006) Optimization of the posterior condylar offset, tibial slope, and condylar roll-back in total knee arthroplasty. J Arthroplasty 21(6):889-896

16. Zhang H, Lü L, Zhang Y, Xu Y, Wang W, Wei B (2011) Influence of posterior condylar offset and anteroposterior femorotibial translation on knee flexion after posterior cruciate-sacrificing self alignment bearing total knee arthroplasty. Zhongguo xiu fu chong jian wai ke za zhi 25(1):42-46

17. Kansara D, Markel DC (2006) The effect of posterior tibial slope on range of motion after total knee arthroplasty. J Arthroplasty 21(6):809-813

18. Kurosaka M, Yoshiya S, Mizuno K, Yamamoto T (2002) Maximizing flexion after total knee arthroplasty: the need and the pitfalls. J Arthroplasty 17(4): 59-62

19. Kim J-H (2013) Effect of posterior femoral condylar offset and posterior tibial slope on maximal flexion angle of the knee in posterior cruciate ligament sacrificing total knee arthroplasty. Knee Surg Related Res 25(2):54

20. Soda Y, Oishi J, Nakasa T, Nishikawa K, Ochi M (2007) New parameter of flexion after posterior stabilized total knee arthroplasty: posterior condylar offset ratio on X-ray photographs. Arch Orthop Trauma Surg 127(3):167-170

21. Li G, Park SE, DeFrate LE, Schutzer ME, Ji L, Gill TJ et al (2005) The cartilage thickness distribution in the tibiofemoral joint and its correlation with cartilage-to-cartilage contact. Clin Biomech 20(7):736-744

22. Johal P, Hassaballa MA, Eldridge JD, Porteous AJ (2012) The posterior condylar offset ratio. Knee 19(6):843-845

23. Kotani A, Yonekura A, Bourne RB (2005) Factors influencing range of motion after contemporary total knee arthroplasty. J Arthroplasty 20(7):850-856

24. Debette C, Lustig S, Servien E, Lording T, Villa V, Demey G et al (2014) Total knee arthroplasty of the stiff knee: three hundred and four cases. Int Orthop 38(2):285-289

\section{Publisher's Note}

Springer Nature remains neutral with regard to jurisdictional claims in published maps and institutional affiliations.

Ready to submit your research? Choose BMC and benefit from:
- fast, convenient online submission
- thorough peer review by experienced researchers in your field
- rapid publication on acceptance
- support for research data, including large and complex data types
- gold Open Access which fosters wider collaboration and increased citations
- maximum visibility for your research: over 100M website views per year
At BMC, research is always in progress.
Learn more biomedcentral.com/submissions

